\title{
The role and effectiveness of partner notification in STD control: a review
}

\author{
Frances M Cowan, Rebecca French, Anne M Johnson
}

\begin{abstract}
Introduction
Partner notification (also known as contact tracing) is the process of contacting the sexual partners of an individual with a sexually transmitted disease (STD) and advising them that they have been exposed to infection. By this means people at high risk of an STD, many of whom are unaware that they have been exposed, are contacted and encouraged to attend for screening and treatment. Partner notification endeavours to reduce the burden of asymptomatic disease in the community and shorten the average period of infectiousness for a given disease, in the expectation that this will reduce disease transmission within the population. It constitutes one aspect of STD control alongside education and screening and treatment of cases. While partner notification has long been accepted as a cornerstone of STD prevention and control, the human immunodeficiency virus (HIV) epidemic has brought with it debate about the ethics, acceptability and effectiveness of partner notification both for the individuals involved and for society in general. In the context of HIV infection the World Health Organisation has defined partner notification as that public health activity in which sexual partners of individuals with HIV infection and those sharing injecting equipment are notified, counselled about their exposure and offered services. ${ }^{1}$ Partner notification is also relevant for injecting drug users who have been in contact with other parenterally transmitted infections including hepatitis $B$ and hepatitis $C$ through sharing needles with an infected person.
\end{abstract}

\section{History}

Partner notification was probably practised for many years before it became formally introduced as a means of STD control in various countries around the world.

Britain In Britain, partner notification was first documented as a method of STD control in the 19th century. The Contagious Disease Acts of 1864 and 1866 were passed due to the increasing incidence of venereal disease amongst the military. ${ }^{2}$ Prostitutes incurred the brunt of control measures which included confinement for up to six months. The Acts were repealed in 1868, after campaigning from social reformers.

The high prevalence of syphilis and gonorrhoea in London in the early 20th century led the Government to establish a Royal Commission whose report in 1916 recommended that free, open access and confidential services should be established for the treatment of STDs. ${ }^{3}$ It was not until 1942, when STD control became regarded as a national emergency, that prevention and in particular tracing of sexual partners was recognised as important in STD control in Defence Regulation 33B. ${ }^{4}$ It recommended that specialised staff should be employed for this work. Although the regulations were repealed in 1947 as it was thought the incidence of gonorrhoea and syphilis would fall during peacetime, the Ministry of Health did recommend that contact tracing procedures should continue.

A network of open-access STD clinics was established following the Royal Commission Report of 1916, providing free confidential service for the diagnosis and treatment of STDs. These clinics are responsible for collecting national statistics on STDs which are reported quarterly to the Department of Health (previously the Department of Health and Social Security). The first official document to outline the contact tracing process and give a standard for good practice was included in the National Health Service [Venereal Diseases] Regulations in $1968 .^{5}$ This was superseded by the National Health Service [Venereal Disease] Regulations 1974, which are still in place today. ${ }^{6}$ These regulations prohibit disclosure of information which could be used to identify an individual who has been examined or treated for a sexually transmitted disease except when it is in the individuals interest or for disease prevention.

The process developed for notifying partners in Britain centred around the use of contact slips. Patients with an STD were given one contact slip for each of their sexual contacts. Information recorded on each contact slip include patient identification number (but not name or address) to allow cross referencing, the date, details of the issuing clinic and a code detailing the patient's diagnosis (the Department of Health developed codes for each of the STDs which were used nationally, allowing the diagnosis of the index case to remain confidential while enabling the physician screening and treating the contact to do so appropriately). The contacts then took the contact slip to their local STD clinic where they were screened and treated. Contact slips should then be returned to the issuing clinic for recording in the index patients notes. Contact slips are still used in many clinics for partner notification of STDs other than HIV. The advantage of this system is that it allows confidentiality of the contact to be preserved. However, the relative effectiveness of using codes on contact slips rather than the actual diagnosis has never been evaluated.

In Britain the majority of partner notification is done by health advisers (previously known as contact tracers) who are attached to 
STD clinics. Over the last decade, the role of the health adviser has evolved in response to the HIV epidemic with counselling, health education and prevention work forming an increasing part of their work. In some clinics this has resulted in a change in emphasis from responsibility to the public health towards responsibility to the individual patient. ${ }^{7}$ Some health advisers have found it difficult to reconcile their counselling role in which they provide non-directive support to individuals with their public health role in partner notification. ${ }^{8}$ There is some evidence that partner notification of non-HIV STDs has deteriorated as a result of this change in emphasis. 9

USA In the US in 1936, Thomas Parran, the architect of the federal anti-venereal disease programme advocated screening, case finding, immediate treatment and contact tracing of people with syphilis as he believed it was only in this way that it would be possible to break "the chain of disease transmission". ${ }^{10}$ In 1938 Congress passed the National Venereal Disease Control Act which leant federal support to STD control programmes. By the 1940s "contact epidemiology" had become a central feature of syphilis control programmes. ${ }^{11}$ Federal support for venereal disease control was cut back during the 1950s and it was not until 1972 that the Public Health Service established a gonorrhoea control programme. ${ }^{11}$

In the US, the majority of partner notification, at least in the public sector, is done by trained interviewers attached to Public Health Departments, who have developed standardised techniques for interviewer training, patient and contact interviewing and for office and field follow-up of patients and contacts in addition to developing information systems capable of local and national monitoring of contact tracing.

Scandinavia Partner notification for STDs has been practised in Sweden since the 19th century. Since 1944, social workers have been attached to STD clinics to assist with contact tracing. ${ }^{12}$ It is a legal requirement for patients with STDs including HIV to notify their sexual partners. ${ }^{13}$ Contacts who do not attend after being notified of their infection risk can be compelled to do so. This contrasts with practice in Denmark where there is strong opposition to partner notification for HIV and confidentiality of the HIV positive patient is absolute. ${ }^{14}$

In many other parts of the world partner notification is very limited in part due to the lack of a formal network of STD clinics.

\section{Definitions}

Partner notification can be performed in a variety of different ways:

patient referral

the index patient is encouraged to contact his or her sexual partners and advise them to seek appropriate medical care. This process can be assisted by health care workers who spend time educating the patient about the importance of contact tracing, give out contact cards or using telephone or mail reminders.

provider referral the health care workers involved in the index patient's care can notify the sexual partners without naming the patient concerned.

conditional referral the health care worker of the index cases obtains names of their sexual partners but allows patients a period of time to notify partners themselves. If the partners are not notified within this time period the health care professional notifies their sexual partners without naming the patient concerned.

One of the fundamental tenets of partner notification is that confidentiality of the source partner is maintained absolutely. The contact is merely informed that they have been exposed to infection and offered screening and treatment for infection as deemed appropriate. In practice, however, if the contact has had only one partner then confidentiality is impossible to preserve. ${ }^{15}$

\section{Theoretical rationale for partner notification}

The distribution of an STD within a community is dependent on both the sexual behaviour of individuals within that community (including rate of partner change, extent of mixing between high and low risk populations and barrier contraceptive usage) as well as the efficiency of transmission and the duration of infectiousness of the STD. ${ }^{16}$ The rationale behind partner notification for bacterial STDs is that individuals who have been exposed to an STD but are asymptomatic can be identified and cured of their infection reducing both their morbidity and duration of infectiousness and thereby breaking the chain of transmission of infection. In this scenario both the individual and the community can be seen to gain from partner notification. Traditionally little emphasis was placed on health education or promoting safer sexual behaviour for bacterial STD control on the basis that this might discourage people from attending STD clinics for screening and treatment.

With the advent of viral STDs including HIV the rationale for partner notification is less clear. Asymptomatic contacts may have less to gain personally as a result of being notified: if infected they cannot be cured of their infection. However, there is evidence to suggest that early identification of asymptomatic HIV infected individuals is worthwhile. For example, pregnant women can reduce their risk of vertical transmission of infection by taking zidovudine during pregnancy and labour. ${ }^{17}$ Early intervention with prophylactic therapy for opportunistic infections such as Pneumo- 
cystis carinii pneumonia and toxoplasmosis has been shown to delay the onset of these infections among HIV infected individuals. ${ }^{18}$ In addition Porter et al have examined the voluntary AIDS notifications for England and Wales and shown that those individuals who were found to be HIV infected shortly before their AIDS diagnosis were more likely to die within a month of diagnosis than those who had known about their diagnosis for sometime. ${ }^{19}$ The association between an increased risk of HIV transmission and late stage of disease also strengthens the argument for earlier identification of HIV infected individuals. ${ }^{20}$

From an epidemiological perspective, partner notification for viral STDs including HIV is only worthwhile if people who become aware of their infectivity modify their behaviour to reduce their risk of further disease transmission. There is limited evidence to suggest that this happens. ${ }^{21}$ For example in the European Study of heterosexual transmission of HIV infection around half of the 245 couples taking part continued to have unprotected sexual intercourse despite repeated counselling. ${ }^{22}$ Therefore for viral STDs there may be less to gain for both the individual and the community than for partner notification of bacterial STDs. Primary prevention (preventing acquisition of infection) either through vaccination or changing sexual behaviour must therefore remain the cornerstone of viral STD control in contrast to secondary prevention (preventing transmission of infection by treatment) for bacterial STDs.

A benefit of partner notification for the contacts of viral STDs is that they can be screened for coincident bacterial STDs for which they may also be at risk. Wooley conducted a retrospective clinical audit on women presenting with initial genital herpes to assess the value of examining their male partners. ${ }^{23}$ In addition to detecting apparent or inapparent genital herpes in around one third of contacts, $19 \%$ of the men were found to have some other lower genital tract infection when they were screened.

\section{Evidence of efficacy of partner notification}

Various process and outcome measures can be used to measure efficacy of partner notification including the number of contacts identified or notified, the number of contacts who presented for screening, the number of contacts identified who tested positive, and the number of contacts who were treated for an STD. The relative cost per contact identified by different strategies can be compared.

There have been many accounts of contact tracing which describe the process used and the number of contacts reached. For example, a scheme in Tyneside pioneered setting up a programme for partner notification and evaluating its outcomes. Staff were employed to interview patients, locate the contacts, visit them and persuade them to attend for treatment. Much energy was put into following up defaulters. Within the first 6 months of the scheme, 241 out of 451 "named" women were identified, of whom 211 attended the clinic and 103 were diagnosed with syphilis and/or gonorrhoea. Wigfield documented continued success at contact tracing over a further 27 years of the scheme. ${ }^{24} \mathrm{Katz}$ et al reported on using field follow up (that is a strategy where clinics assumed the responsibility to assure all partners attended for examination) to locate contacts of 12732 heterosexual patients with gonorrhoea, chlamydia or related syndromes between 1983 and 1989 in Indianapolis. ${ }^{25} \mathrm{~A}$ "disease intervention specialist" was able to locate $82 \%$ of their 13845 named contacts during that period. In a study on partner notification for Chlamydia trachomatis from rural Nova Scotia 25 of 37 were contacted. ${ }^{26}$ Giesecke and colleagues evaluated a Swedish partner notification programme for HIV infection over an 18 month period during 1989-90. ${ }^{27}$ A group of $365 \mathrm{HIV}$ positive patients identified 564 sexual or needle sharing contacts, of whom 390 were located and had pre HIV test counselling. HIV antibody results were established for 350 of these contacts. Overall 53 new cases of HIV infection were diagnosed.

It appears that partner notification is a relatively ineffective means of disease control when sex with anonymous partners (such as prostitutes) is common or when there is likely to be considerable delay before contacts can be traced. A study of an outbreak of heterosexually acquired syphilis associated with crack use in Oregon in 1987 suggested that contact tracing failed as an isolated control measure. ${ }^{28}$ A descriptive study performed in Harare suggested that provider referral was labour intensive and yielded very poor returns with only 20 contacts located in 3 months by six members of staff. ${ }^{29}$ Likewise 141 letters sent to contacts of patients seen in Ibadan, Nigeria only resulted in seven people attending for screening. ${ }^{30}$ In this circumstance other public health interventions such as mass screening or treatment programmes may prove to be more efficacious in reducing the burden of disease within the population. ${ }^{29}$ Partner notification is also likely to be ineffective if health services are either inaccessible or unacceptable to clients.

While descriptive studies give useful accounts of the different contact tracing procedures they are of limited value in assessing the comparative efficacy of one approach compared with another.

Oxman and colleagues have recently performed a systematic overview of the biomedical literature assessing the efficacy of alternative partner notification strategies for STDs (gonorrhoea, chlamydia, syphilis, HIV and hepatitis B). ${ }^{31}$ Following a comprehensive search for both published and unpublished studies, using a combination of electronic and hand searching plus personally contacting 80 key informants, they were able to identify 12 comparative studies which met their criteria for inclusion. (Inclusion criteria were: studies with target population which consisted of partners of patients with gonorrhoea, chlamydia, syphilis, HIV infection or hepatitis B in which at least two partner notification strategies were compared and any of the following outcome 
measures were recorded-partners were identified, notified, presented for care, tested positive or were treated, costs or ethical consequences.) Seven of the eligible studies provided data on the referral process, ${ }^{32-38}$ four provided comparison of trained interviewers with routine care providers; ${ }^{3639-41}$ and three evaluated patient assistance aimed at facilitating patient referral..$^{37243}$ Five of these studies were methodologically strong, ${ }^{33} 36374243$ three were moderately strong 343840 and there were serious threats to the validity of four studies. $^{32353941}$ The authors were only able to draw limited conclusions on the effectiveness of different approaches on the basis of the available data. Their conclusions are summarised here. 1) There is strong evidence that simple forms of patient assistance directed at improving patient referral can be effective. Three randomised controlled trials (RCTs) have examined whether the results of patient referral can be improved by simple measures such as showing the index case an educational video, ${ }^{42}$ using referral card with telephone follow-up of index patient ${ }^{43}$ and interviewing index patients rather than giving out an information leaflet on importance of partner notification (unpublished study). These interventions were shown to be relatively effective and cheap. Telephone follow-up for example cost US $\$ 2 \cdot 24$ per partner traced.

2) There is moderately strong evidence that provider referral results in more patients being notified than patient referral for HIV infection. One RCT has compared provider referral with patient referral for HIV positive patients. ${ }^{38}$ This demonstrated that only two index patients would have to be offered provider referral for one additional partner to be notified. Of note, $94 \%$ of the 88 notified were not aware that they had been exposed to HIV infection. A further RCT examining four different approaches to partner notification in HIV is currently underway.

3) There is weak evidence that provider or conditional referral is more effective than patient referral for syphilis. This is based on the results of a study conducted in Arkansas in 1948 which compared the results of intensive provider referral and field notification with routine provider referral for syphilis over two different time periods ${ }^{32}$ and a before and after study from Poland where all index patients were re-interviewed to see if partner notification had occurred. ${ }^{39}$

4) Conflicting evidence exists regarding the effectiveness of provider and conditional referral compared with patient referral for gonorrhoea and chlamydia. Two studies have compared conditional/provider referral with patient referral for gonorrhoea and have reported conflicting results. One found no difference between the two approaches ${ }^{33}$ while the other showed a benefit from conditional referral (conditional referral would need to be offered to eight index patients to identify one additional culture positive partner). ${ }^{37}$ An RCT which compared provider with patient referral for patients with non-gonococcal urethritis found that provider referral was more effec- tive $^{36}$ and that provider referral would have to be offered to only two patients with chlamydia for two additional contacts to be assessed. Conditional/provider referral was 4-8 times more expensive than patient referral in these studies.

5) There is weak evidence that trained interviewers are more effective than routine health care workers at identifying partners, but no evidence that this results in practically important benefits. ${ }^{3741}$

These studies have all been carried out using different methods, in different cultures and health care systems. All are relatively small and show conflicting results for different diseases. This may be as much to do with different interventions and study design as it is to the different diseases. There is still an important place for further randomised controlled trials in this area. Strikingly absent from the literature are any community-based comparison studies which attempt to evaluate the effectiveness of partner notification in reducing the incidence/prevalence of disease in the community.

\section{Acceptability}

In order to evaluate the partner notification process, acceptability for the index patient, the contact and the staff involved in the process has been assessed, in a small number of studies.

Index case and contacts Acceptability seems to be determined by two factors, maintenance of confidentiality and availability of treatment. An unacceptable strategy for partner notification could potentially jeopardise the relationship between health care workers and their clients and may succeed in discouraging infected individuals to seek care. Most of the data on acceptability are anecdotal and in relation to partner notification for HIV infection. A study of 25 women with HIV infection in New Jersey showed that $68 \%$ of them were willing to give names of their sexual partners to the Health Department as long as their confidentiality would be maintained, whereas only $20 \%$ would agree to partner notification if their names were disclosed to the partner. ${ }^{44}$ In practice though, only $24 \%$ of the women had informed partners they had had previous to their HIV positive diagnosis and $52 \%$ had informed partners they had had since their diagnosis. A study in South Carolina administered an anonymous, self-completion questionnaire to 132 partners of HIV infected patients, all of whom were informed of their exposure to HIV infection by the public health department. ${ }^{45}$ When asked if they thought the public health department did the right thing in telling them of their risk, $87 \%$ responded "yes". When asked if partner notification by the public health department should continue 97\% responded "yes".

Acceptability may vary between populations. Pavia et al found in their evaluation of the Utah Partner Notification Programme for HIV infection, that HIV partner notification was less successful in white homosexual and bisexual men compared with other groups. ${ }^{46}$ 
They concluded that this may be due to greater distrust of public health authorities and that homosexual and bisexual men preferred to notify partners without the help of public health workers. In a population well educated about HIV infection and its transmission, partner notification may not be an effective strategy as individuals may be aware of their risk and have made a conscious decision not to be tested. Poulin et al in their evaluation of a partner notification programme for hepatitis B in injecting drug users, noted that desire for more information and the availability of a vaccine, probably assisted cooperation and acceptance. ${ }^{47}$

Assessing the acceptability of partner notification for contacts and index cases is further complicated by the fact that it may vary according to when it is carried out, in that it seems likely that the contacts may feel differently about the process immediately after being notified and six months after the event.

Staff A successful partner notification programme will only work if it is acceptable to the staff involved. Prior to the HIV epidemic, the acceptability of partner notification to health care workers was not an issue. The long incubation period and lack of cure, plus the emotional distress and prejudice experienced by those who are HIV positive, has caused the profession to debate the benefits of partner notification for HIV infection. Allen and Hogg in their research for the UK Policy Studies Institute compared the views of doctors, nurses and health advisers on partner notification for HIV infection and for all other STDs. The information was gained through interviews with staff from 20 genitourinary clinics in England. They found that $22 \%$ of doctors, $18 \%$ of the nurses and $5 \%$ of the health advisers thought that partner notification for HIV infection must be done. This compared with $67 \%$ of the doctors, $66 \%$ of the nurses and $55 \%$ of the health advisers who thought that partner notification must be done for other STDs. Twenty two per cent of the doctors, $18 \%$ of the nurses and $40 \%$ of the health advisers would encourage patients to inform their partners of their HIV risk themselves, but on a voluntary basis. Keenlyside et al found that HIV partner notification was discussed

Summary of proposed practice guidelines for partner notification ${ }^{49}$

\footnotetext{
As a principle all sexual and needle sharing partners of patients with an STD should be informed of their exposure.

Evidence to date does not reliably indicate the relative effectiveness of different strategies, therefore the minimum intervention should be patient referral.

There is not enough evidence to suggest that different strategies should be implemented for different STDs therefore the recommendations below apply to partner notification for all STDs.

1. Training of health care providers to enhance counselling and risk assessment of patients and approach to partner notification.

Health care providers should be able to give assistance to patients requesting help with partner referral.

Index patients should be followed up within a few weeks to assess success of patient referral and to offer further assistance.

If necessary, legislation should be introduced to allow mandatory provider referral in certain well defined circumstances.

Mandatory naming of partners as an initial part of any partner notification process should be discouraged.

The following recommendations are based on expert opinion

6 Notification of sexual partners for hepatitis B should be done by staff trained in

portner notification rather than those trained in enteric diseases.
partion trained in

Public health departments should examine process data on partner notification to enhance management decisions about them.
}

with the majority of patients. Fifty five per cent of doctors and $67 \%$ of health advisers would routinely discuss partner notification during pre-test counselling and $98 \%$ of doctors and $81 \%$ of health advisers would, during post-test counselling, encourage those patients found to be HIV positive to get in touch with partners who may be at risk. ${ }^{48}$ There was marked variation between centres. Centres with large caseloads within London were less likely to discuss partner notification than the smaller centres without. However, partner notification may be relatively more important in the smaller centres serving low prevalence communities where the population may not have personalised their risk of infection. It is striking that the individuals who are employed partly for their role as partner notifiers are those less likely to support partner notification for HIV.

These studies suggest a marked geographical and inter-disciplinary difference in opinion.

Ethics There has been much debate on the ethics of partner notification over the last decade. The controversy surrounding partner notification of viral STDs in general and HIV in particular has focused attention on the polemic between the rights of the individual to absolute confidentiality versus the rights of society to be warned of any risk of infection and to take measures to reduce spread through the community. This controversy remains unresolved with different countries employing strategies which vary from societal to individual in approach. Some countries have chosen to legislate to enforce their approach while others have supported an agreed code of practice. Blaxter in her overview of worldwide prevention and control of HIV describes the different approaches to partner notification taken around the world, ranging from Denmark and Norway where confidentiality of the index patient is absolute and there is opposition to "official" partner notification, to Iceland and Sweden, where contacts are legally bound to come forward for testing. ${ }^{14}$

Guidelines Millson et al have published a proposal for guidelines for partner notification of STDs. ${ }^{49}$ These guidelines were developed using various sources of evidence including that from the systematic overview of partner notification by Oxman et $a l^{31}$ in addition to the results obtained from a nationwide survey of current Canadian practice and using advice from experts in the field. ${ }^{50}$ These proposed guidelines are outlined in the table.

In the UK recommendations for partner notification are detailed in a Health Education Council guide Handbook on contact tracing in STDs. ${ }^{51}$ This does not currently contain any recommendations for partner notification of either chlamydia or HIV infection and is being updated.

The Centers for Disease Control in the US includes disease specific recommendations for partner management as part of their treatment guidelines. $^{52}$ 


\section{Conclusion}

Partner notification has been an important component of STD control for at least the last 40 years; however, the rationale behind it is likely to have been altered by the relative shift from bacterial curable STDs to non-curable viral STDs over the last 15 years. Although there is evidence that asymptomatic cases of STD are identified as a result of partner notification, the societal benefits and relative cost effectiveness of different strategies are still poorly understood. There has been little work to date to demonstrate a direct effect of partner notification on the incidence/prevalence of STDs within the community. It seems likely that the emergence of the HIV epidemic may have detracted from the efficacy of partner notification for traditional STDs in some countries. What is needed now are randomised controlled trials designed to examine the comparative efficacy and cost effectiveness of different strategies for partner notification in addition to studies which further examine its acceptability to patients, contacts and staff.

1 WHO consultation document, WHO/GPA/ESR/89.2

2 Adler MWA. The terrible peril: a historical perspective on the venereal diseases. BMF 1980;281:206-11.

3 Royal Commissioners on Venereal Diseases. Final report of the commissioners. London HMSO, 1916. (Cmnd 8189).

4 House of Lords Parliamentary Debates. Official Report. 125, No 435. London: HMSO, 1942.

5 National Health Service, England and Wales (Venerea Disease) Regulations $1968 \mathrm{HM}(68) 84$

6 National Health Service, England and Wales (Venerea Diseases) Regulations 1974 No 29 [H 334]

7 Allen I, Hogg D. Work roles and responsibilities in genitourinary medicine clinics. London: Institute for Policy Studies, 1993.

8 Bond T. HIV counselling: report on national survey and consultation. British Association for Counselling/ consultation. British Association for

9 Murray J. An audit of partner notification for gonorrhoea and chlamydia (unpublished).

10 Parran T. The next great plague to go. Survey Graphic 1936;25:405-11.

11 Brandt AM. No magic bullet-a social history of venerea disease in the United States since 1880. Expanded Edition. Oxford University Press; 1987, New York.

12 Rudén, Jonsson A, Lidbrink P, Allebeck P, Bygdeman SM. Endemic versus non-endemic gonorrhoea in Stockholm: results of contact tracing. Int 7 STD AIDS 1993;4: 284-92.

13 Thelin I, Wennstrom A-M, Mardh P-A. Contact-tracing in patients with genital chlamydial infection. $\mathrm{Br} \mathcal{F}$ Venereal patients with genital
Dis 1980;56:259-62.

14 Blaxter M. AIDS: Worldwide policies and problems: London. Office of Health Economics, 1991.

15 Bayer R, Toomey EK. HIV prevention and the two faces of partner notification. Am f Pub Health 1992;82:1158-64.

16 Hethcote HW, Yorke JA. Gonorrhoea: transmissio dynamics and control. Biomathematics 1984;56:1-105.

17 Connor EM, Sperling RS, Gelber R, et al. Reduction of maternal-infant transmission of human immunodeficiency virus type 1 with zidovudine treatment. $N$ Engl $f$ Med 1994;331:1173-80.

18 Oskenhendler E, Charreau I, Tournierie C, Azihary M, Carbon C, Aboulker IP. Toxoplasma gondii infection in advanced HIV infection. AIDS 1994;8:483-7.

19 Porter K, Wall PG, Evans BG. Factors associated with lack of awareness of HIV infection before diagnosis of AIDS BMf 1993;307:20-22.

20 Johnson AM. Condoms and HIV transmission. N Engl Med 1994;331:391-2.

21 Cleary PD, Van Devanter N, Rogers TF, et al. Behaviour changes after notification of HIV infection. Am $\mathcal{F}$ Public

22 de Vincenzi I. A longitudinal study of human immunodefi- ciency virus transmission by heterosexual partners. $N$ Engl f Med 1994;331:341-6.

23 Wooley PD. Value of examining the sexual partners of women suffering from initial genital herpes. Int $\mathcal{f} S T D$ and AIDS 1991;2:365-6.

24 Wigfield AS. 27 years of uninterrupted contact tracing. The "Tyneside Scheme". B f Venereal Dis 1972;48:37-50.

$25 \mathrm{Katz}$ BP, Caine VA, Jones RB. Evaluation of field followup in a sexually transmitted disease clinic for patients at risk for infection with neiserria gonorrhoea and chlamyrisk for infection with neiserria gonorrhoea and

26 Langille DB, Shoveller J. Partner notification and patien education for case of chlamydia trachomatis infection in rural Nova Scotia. Can $\mathcal{F}$ Pub Health 1992;83:358-61.

27 Gieseke J, Ramstedt K, Granath F, Ripa T, Rådö G Westrell $M$. Efficacy of partner notification for HIV infection. Lancet 1991;338:1096-9.

28 Andrus JK, Fleming DW, Harger DR, et al. Partner notification: can it control epidemic syphilis? Ann Intern Med 1990;112:539-43.

29 Grosskurth H, Plummer F, Mhalu F, Mabey D. STD research in Africa. Lancet 1993;342:1415-6.

30 Asuzu MC, Rotowa NA, Ajayi IO. The use of mail reminders in STD contact tracing in Ibadan, Nigeria. East African Medical fournal 1990;67:75-6.

31 Oxman AD, Sillson ME, Rasoly I, et al. Partner notification for sexually ME, Rasoly I, et al. Partner notification for sexually Public Health 1994;Suppl 1:S41-7.

32 Ensley EJ, Parkhurst GE, Weank RR. The 100 day experiment in contact investigation in Arkansas. Fournal of Venereal Disease Information 1948;29:13-9.

33 Potterat J, Rothenburg R. The case finding effectiveness of a self referral system for gonorrhoea: a preliminary report. Am F Pub Health 1977;67:174-6.

34 Judson FN, Wolf FC. Tracing and treating contacts of gonorrhoea patients in a clinic for sexually transmitted diseases. Public Health Rep 1978;93:460-3.

35 Woodhouse DE, Potterat JJ, Mutch JB, et al. A civilian military partnership to reduce the incidence of gonorrhoea. itary partnership to reduce the incic
Public Health Rep 1995;100:61-5.

36 Katz BP, Danos CS, Quinn TS, et al. Efficiency and costeffectiveness of field follow-up for patients with chlamyeffectiveness of field follow-up for patients with chlamydia trachomatis infection in a sexually

37 Clevland (unpublished) Randomised controlled trials of partner notification strategies for gonorrhoea. 1992.

38 Landis SE, Schoenbach VJ, Weber DJ, et al. Results of randomised trial of partner notification in cases of HIV infection in North Carolina. N Engl f Med 1992;326: $101-6$.

39 Capinski TZ, Urbanczyk J. Value of re-interviewing in contact tracing. Br F Venereal Dis 1970;46:138-40.

40 Hammar H, Ljungberg L. Factors effecting contact tracing of gonorrhoea. Acta Derm Venereol (Stokh) 1972;52:233-40.

41 Alary M, Joly JR, Poulin C. Gonorrhoea and chlamydia infection: comparison of contact tracing performed by infection: comparison of contact tracing performed by $1991 ; 82: 132-4$.

42 Solomon MZ, Dejong W. The impact of a clinic based educational videotape on knowledge and treatment behaviou of men with gonorrhoea. Sex Transm Dis 1988;15:127-32.

43 Montesinos L, Frisch LE, Greene BF, Hamilton M. An analysis of and intervention in the sexual transmission of disease. F Appl Behav Anal 1990;23:275-84.

44 Chervenak JL, Weiss SH. Sexual partner notification: attitude and actions of HIV-infected women. Presented at V International Conference on AIDS, Montreal, June 8 , 1989. Abstract Th.D.P.4. p 759 .

45 Jones JL, Wycoff RF, Hollis SL, et al. Partner acceptance of Health Department notification of HIV exposure, South Health Department notification of

46 Pavia AT, Benyo M, Niler L, Risk I. Partner notification for control of HIV: results after 2 years of a statewide program in Utah. Am ₹ Public Health 1993;83:1418-24.

47 Poulin C, Gyorkos TW, MacPhee J, Cann B, Bickerton J. Contact tracing among injection drug users in a rural area. Can $\mathcal{F}$ Health 1992;83:106-8.

48 Keenlyside RA, Hawkins AS, Johnson AM, Adler MW. Attitudes to tracing and notifying contacts of people with HIV infection. BMF 1992;305:165-8.

49 Millson ME, Rasoly I, Scott EAF, et al. Partner notification for sexually transmitted diseases: proposed practice guidelines. Can ₹ Pub Health 1994;Suppl 1:S53-5.

50 Rasoly I, Millson ME, Frank JH, et al. A survey of public health partner notification for sexually transmitted diseases in Canada. Can $f$ Pub Health 1994;Suppl 1: eases in

51 Hunter I, Jacobs J, Kinnel H, Satin A. Handbook on contact tracing in sexually transmitted diseases. London: The Health Education Council, 1980: (The Health Education Council Ed).

52 CDC Sexually transmitted diseases treatment guidelines. Centers for Disease Control, Atlanta 1993 